\title{
Executive Functioning in Cognitively Normal Middle-Aged Offspring of Late-Onset Alzheimer's Disease Patients
}

Carolina Abulafia, B.A. ${ }^{1-4}$, Leticia Fiorentini, Ph.D. ${ }^{1,2,4}$, David A. Loewenstein, Ph.D. ${ }^{5}$, Rosie Curiel-Cid, Psy.D5 , Gustavo Sevlever, M.D., Ph.D.6,7, Charles B. Nemeroff, M.D., Ph.D. ${ }^{8}$, Mirta F. Villarreal, Ph.D. ${ }^{1,2,9}$, Daniel E. Vigo, M.D., Ph.D. ${ }^{2,3,10}$, Salvador M. Guinjoan, M.D., Ph.D. ${ }^{1,2,4,11}$

${ }^{1}$ Grupo de Investigación en Neurociencias Aplicadas a las Alteraciones de la Conducta. FLENI, Buenos Aires, Argentina

${ }^{2}$ National Council for Scientific and Technological Research (CONICET), Argentina

${ }^{3}$ Institute for Biomedical Research (BIOMED), School of Medical Sciences, Universidad Católica Argentina, Buenos Aires, Argentina

${ }^{4}$ Servicio de Psiquiatría. FLENI, Buenos Aires, Argentina

${ }^{5}$ Department of Psychiatry and Behavioral Sciences and Center on Aging, Miller School of Medicine, University of Miami, United States of America

${ }^{6}$ Departamento de Neuropatología y Biología Molecular. FLENI, Buenos Aires, Argentina 
${ }^{7}$ Departamento de Docencia e Investigación. FLENI, Buenos Aires, Argentina

8 Institute of Early Life Adversity Research, Dell Medical School, The University of

Texas, Austin, United States of America

${ }^{9}$ Department of Physics (FCEyN), University of Buenos Aires, Argentina

${ }^{10}$ School of Psychology and Educational Sciences, Katholieke Universiteit Leuven, Belgium

11 Departamento de Psiquiatría y Salud Mental, Unidad docente FLENI, Universidad de Buenos Aires, Facultad de Medicina, Argentina

\section{Corresponding author}

Salvador M. Guinjoan

Service of Psychiatry, FLENI Foundation

Montañeses $23255^{\text {th }}$ floor

C1428AQK Buenos Aires

Argentina

sguinjoan@fleni.org.ar

+541157773200 x2514/2531 


\section{Abstract}

Episodic memory deficits are traditionally seen as the hallmark cognitive impairment during the prodromal continuum of late-onset Alzheimer's disease (LOAD). Previous studies identified early brain alterations in regions subserving executive functions in asymptomatic, middle-aged offspring of patients with LOAD (O-LOAD), suggesting that premature episodic memory deficits could be associated to executive dysfunction in this model. We hypothesized that O-LOAD would exhibit reduced executive performance evidenced by increased errors and decreased strategy use on an episodic memory task. We assessed 32 asymptomatic middle-aged O-LOAD and 28 age-equivalent control subjects (CS) with several tests that measure executive functions and the Rey Auditory Verbal Learning Test (RAVLT) to measure memory performance. All tests were scored using both traditional and process scores (quantification of errors and strategies underlying overall performance). T-tests were used to compare performance between both groups and Spearman correlations were implemented to measure associations between variables. O-LOAD participants exhibited decreased executive performance compared to CS as it relates to initiation time (Tower of London), mental switching (Trail Making Test B), and interference effects (Stroop Word-Color condition). Traditional RAVLT measures showed a poorer performance by O-LOAD and RAVLT process scores revealed increased interference effects on this group. Positive correlations $\left(r_{s}\right)$ were found between the executive measures and several RAVLT measures for O-LOAD but not for CS. In conclusion, O-LOAD participants exhibited early subtle cognitive changes in executive processing. Observed 
memory difficulties may be associated in part to executive deficits suggesting an interplay between memory and executive functions. Process score impairments were observed earlier than clinical decline on neuropsychological scores in this atrisk cohort and might be useful cognitive markers of preclinical LOAD.

Keywords: Preclinical late-onset Alzheimer's disease, RAVLT, executive functions, process scores, subtle cognitive changes 


\section{Introduction}

The term "executive functions" (EF) is not a unitary concept, but rather is an umbrella term used for various complex cognitive processes that are critical for engagement in complex thought and behavior (Hazlett et al., 2015). EF include numerous cognitive processes (i.e. planning, goal selection, set-shifting, working memory, self-monitoring, inhibition of inappropriate behavioral responses) associated with different regions within the frontal lobes - particularly the prefrontal cortex and the anterior cingulate - and are distributed throughout a wide network that includes parietal regions, subcortical structures and thalamic pathways (Jurado and Rosselli, 2007).

EF have been recently receiving growing attention in the Alzheimer's disease (AD) literature. Specifically, deficits within this cognitive domain are associated with decreased functioning in activities of daily living, which represent an essential diagnostic criterion for a clinical diagnosis of AD (Hazlett et al., 2015). The presence of executive impairment is well established in mild and moderate AD (Albert, 1996; Binetti et al., 1996; Swanberg et al., 2004; Kirova et al., 2015; Blanco Martín et al., 2016) and, progressively, more authors are suggesting an early impairment in this cognitive domain which is usually present in prodromal $A D$ and even in preclinical stages (Rapp and Reischies, 2005; Blacker et al., 2007; Johns et al., 2012; Reinvang et al., 2012; Ballard and O'Sullivan, 2013). Some investigators have even postulated that attention/executive mild cognitive impairment $(\mathrm{MCl})$ is not a separate subtype of $\mathrm{MCl}$ but may be a precursor to amnestic $\mathrm{MCl}$ in those at risk for $\mathrm{AD}$ and thus, patients with nonamnestic 
attention/executive impairment may develop memory problems later on (Reinvang et al., 2012).

Another reason for increasing interest in EF is that many of the cognitive tests that are most helpful in predicting which nondemented elderly will subsequently develop dementia include substantial executive control requirements (Brandt et al., 2009), specifically, tests assessing episodic memory which is the most common and salient cognitive domain affected in AD (Baudic et al., 2006). A connection between memory and executive impairment in prodromal $A D$ has been recently suggested (Hazlett et al., 2015) and some authors even propose that the early executive dysfunction may contribute to the upcoming memory deficit (Reinvang et al., 2012). There is evidence for statistically significant poorer performance of amnestic $\mathrm{MCl}$ patients on several tests of executive function (Johns et al., 2012; Zheng et al., 2012) and executive improvement derived from cognitive stimulation focused on $\mathrm{EF}$ on $\mathrm{MCl}$ patients generalized to memory performance (Moro et al., 2015). The relationship between EF and memory impairment has not been thoroughly studied and may yield new insights regarding the neurobiology of $\mathrm{MCl}$ and preclinical AD (Libon et al., 2011). In fact, in the absence of hippocampal atrophy in healthy asymptomatic middle-aged offspring of late-onset AD (O-LOAD), our team observed decreased cortical thickness, abnormal cerebral metabolism, and incipient differences in amyloid deposition in precuneus, posterior cingulate, and prefrontal and temporal areas potentially associated with executive functioning rather than with episodic memory (Duarte-Abritta et al., 2018).

Most available studies address $\mathrm{EF}$ in either $\mathrm{MCl}$ or elderly populations while there 
is a paucity of data on middle-aged samples with increased genetic risk of developing AD. In this context, Reinvang and his team (2012) propose that the search for cognitive markers in very early $A D$, that is, even prior to $\mathrm{MCl}$ should focus on subtle cognitive changes. Such changes may be as relevant as other preclinical AD markers (Thomas et al., 2018) and some researchers suggest that these subtle changes are as sensitive as, and sometimes superior to, neurobiological biomarkers at predicting progression to $\mathrm{MCl}$ and subsequently to AD-type dementia (Salmon and Bondi, 2009; Edmonds et al., 2015; Thomas et al., 2018). Given that traditional global neuropsychological scores may be insensitive to the aforementioned subtle changes (Loewenstein et al., 2018b), a recommended approach to detect subtle cognitive decline is the implementation of a process scoring system, that is, the quantification of errors and strategies of an individual that could either hinder or aid performance as a means to determining how and why a final outcome (traditional score) was achieved and allows for a deeper understanding of the brain-behavior relationships underlying all cognitive functioning (Kaplan, 1988). Additionally, process scores are indicative of executive processing involved in other cognitive functions (Lezak et al., 2004). Along these lines, progressively more researchers have been implementing process-approach analysis to assess populations with increased risk of developing AD. Studies on memory tasks found reduced learning slope, increased intrusion errors and increased susceptibility to interference (Libon et al., 2011; Curiel et al., 2013; Sánchez et al., 2017; Abulafia et al., 2018; Thomas et al., 2018). Similar analyses have been also applied to EF tasks evidencing increased commission errors on tests of inhibition and cognitive flexibility (Wetter et al., 2005) as well as 
asymmetric performance on fluency tasks (Houston et al., 2005). Very limited work has been conducted to assess EF abilities in healthy, young at-risk individuals. Recent findings suggested that EF were sensitive to subtle differences between cognitively intact elders with and without family history of AD (Donix et al., 2012) and between APOE4-carrying elders and non-carriers (Reinvang et al., 2010).

In the present study, asymptomatic and cognitively normal middle-aged O-LOAD participants were assessed with widely-used traditional tests of EF and then performed a process scoring system. The same analysis was applied to an episodic memory task extensively used to assess memory complaints, the Rey Auditory Verbal Learning Test (RAVLT), as a way to detect executive processes involved in eventual memory deficits. We hypothesized that O-LOAD would exhibit a reduced performance on EF tests together with increased errors and lower use of strategies. Further, we hypothesized that at this early stage of putative disease, any episodic memory deficits would be related to executive function abnormalities possibly related to encoding, retrieval, self-monitoring and/or inhibitory processes. Regarding memory performance, we predicted increased intrusion and interference errors on instances measuring both proactive and retroactive interference, and on delayed recall trials in O-LOAD when compared to CS.

\section{Materials and Methods}

\section{Design and Sample}


An exploratory cross-sectional study was performed to compare cognitive measures between a sample of 32 offspring of late onset Alzheimer's disease patients (O-LOAD) and 28 control subjects with no family history of $A D(C S)$. Both groups were comparable in age, gender, education level, and depressive symptoms. All participants provided their written informed consent for the study as approved by the local bioethics committee and in accordance with the Declaration of Helsinki.

The inclusion criteria for O-LOAD were as follows: (1) having at least one parent diagnosed with probable LOAD according to the DSM-5 criteria, (2) to be 40-65 years old at the time of recruitment, (3) having seven or more years of formal education, (4) Mini Mental State Examination (MMSE) score >26 (Folstein et al., 1975), (5) no evidence of current progressive neurologic disease or medical conditions likely to impair cognitive function, (6) no history of substance abuse (alcohol, marijuana, stimulants, benzodiazepines, cocaine, or other illicit drugs), and (7) Hachinski score $<4$ to screen out subjects with potential vascular disease (Rosen et al., 1980).

All participants were asked to fill in names, dates of birth, age of AD onset, and clinical information of all affected family members. The information was confirmed with other family members and by interview with the examining physician, discussing the parents' symptomatology and progression of disease. For individuals who had received no treatment at FLENI Foundation the parents' diagnosis of LOAD was certified by a trained clinician from the institution. Only 
individuals whose parents met DSM- 5 criteria for probable AD and had lived to age $\geq 65$ were included.

The volunteers on the CS group had the same inclusion and exclusion criteria described above but were required to have no family history of neurodegenerative disease.

\section{Cognitive Assessment}

The neuropsychological tests included in this study have been widely validated and are frequently used in clinical practice and thus require no detailed explanation. For a thorough study of executive functions the following tests were selected:

Backwards Digits subtest from WAIS III (working memory) (Weschler, 1997); the Trail Making Test (TMT) (Part B for cognitive flexibility and set-shifting) (Reitan and Wolfson, 1985); Design Fluency (DF) from the D-KEFS (nonverbal productivity, inhibition and set-shifting) (36); Phonetic Fluency (letter P) (verbal productivity) (Spreen and Benton, 1977); Similarities subtest from WAIS III (abstract thinking) (Weschler, 1997); Tower of London DX (TOL) (planning and problem-solving) (Culbertson and Zillmer, 2001); and Stroop Test (inhibition on the Word-Color trial) (Golden, 1978). Finally, the Rey Auditory Verbal Learning Test (RAVLT) (Rey, 1964; Schmidt, 1996) - which assesses verbal episodic memory - was incorporated to study the test's inherent executive processes in detail as described below.

To ensure all participants were cognitively asymptomatic, a complete neuropsychological battery was implemented to assess all cognitive domains. The 
individuals' neuropsychological performance was within normal limits on all administered tests according to local norms, and thus none of the individuals met clinical criteria for mild cognitive impairment or dementia, nor did they exhibit subjective cognitive complaints.

The BDI-II questionnaire (Beck et al., 1996) was administered to screen for presence and severity of reported depressive symptoms which could impact cognitive performance. In our sample three CS and seven O-LOAD scored for mild depressive symptoms, but did not fulfill criteria for major depression. No impact on cognitive function was observed in these subjects. To estimate premorbid intelligence the WAT-BA test was administered and an estimated IQ was derived (Sierra Sanjurjo et al., 2015). All tests were administered and scored by a trained neuropsychologist blind to the participant's group (CA).

\section{Process approach scoring analysis}

Additionally to standardized objective scoring, a process scoring analysis was performed on the RAVLT and EF tasks to measure both the amount and type of errors as well as use of strategies that could either hinder or aid performance respectively.

Trail Making Test. The TMT B-A variable is a very common derived score of the TMT which calculates the difference between Part B and Part A. This derived score removes the speed component and reduces the visuoperceptual, motor and attentional demands of the TMT task which gives way to a purer indicator of 
cognitive flexibility and executive control processes (Christidi et al., 2015).

Additionally, errors on the TMT B were quantified and classified as Sequence

Errors when subjects broke either the number sequence (e.g., $8-\mathrm{H}-10$ ) or the letter sequence (e.g., F-7 - I) with preserved number-letter switching. Instances where subjects failed to alternate between numbers and letters were classified as Switching Errors.

Design Fluency. For each of the three conditions (1: Basic, 2: Filter, and 3: Switch) of the task, perseverative errors (Perseverations) were quantified whenever a design was repeated. Switching Errors were reported whenever subjects failed to alternate between empty and filled dots on the Switch condition. Moreover, use of a strategy for a more efficient performance was quantified. To our best knowledge, no strategies were delimited for the D-KEFS Design Fluency; therefore, we opted to implement the strategy criteria developed by Vik and Ruff (1988). The authors recognize the use of a strategy whenever three or more consecutive designs (strategic cluster) reflect a systematic rotation of either the whole design (including mirrored designs) or just a portion of it while the rest of the drawing remains fixed, Perseverative designs are not included as part of a strategic cluster if the original design has already appeared in that same cluster. However, the perseverative design can be included in a subsequent cluster. Given that most subjects generated no more than one cluster per condition (if any) this variable was dichotomized as presence/absence of at least one strategic cluster.

Verbal Fluency. Qualitative analysis of phonemic fluency (PF) was carried out following the guidelines developed by Troyer, Moscovitch and Winocur (1997). 
Based on the concept of clusters, which are defined as groups of consecutive words that belong to a common semantic or phonemic subcategory, they propose two indicators of strategy use. Clustering (mean cluster size) involves effective word retrieval mechanisms from an internal word store. Switching (the number of transitions between clusters) reflects executive processes such as strategic search processes, cognitive flexibility and set-shifting. Besides strategies, perseverative responses were reported.

Stroop. We have quantified the incorrect responses as an additional measure of inhibitory control and self-monitoring. Spontaneous self-corrections were not recorded for this variable.

RAVLT. Besides traditionally reported variables of acquisition and recall, we measured performance errors throughout the task to assess self-monitoring: intrusions and repetitions throughout the learning slope (trials 1-5), List B, Trial 6 and Delayed Recall, and only intrusions (not belonging to List B) on Recognition. To measure susceptibility to proactive interference List B interference errors (words belonging to List A) were recorded and List B performance was compared to Trial A1. For susceptibility to retroactive interference, interference errors (words belonging to List B) were quantified on Trial A6 and on Delayed Recall; and performance on A6 was compared to A5. Additionally, false positive responses belonging to List $B$ on the Recognition trial (Recognition List $B$ E) were recorded as indication of alterations related to source memory (Strauss et al., 2006). 


\section{Statistical Analysis}

Differences between groups were calculated using a t-test for independent samples for continuous variables. Chi-square tests were used for categorical variables. Correlations were evaluated using Spearman correlation coefficients.

Exploratory studies in early stages such as the present project, aim at searching new hypothesis (Jaeger and Halliday, 1998) and thus minimizing Type II errors is strongly recommended (Reiner et al, 2003; McDondald, 2014). Therefore, an FDR correction at $\mathrm{q}=0.2$ was applied to multiple intergroup comparisons and the significance level of two-tailed tests was set at $\alpha=0.05$. All statistical analyses were performed using the SPSS version 22.0 software (SPSS Inc.).

\section{Results}

Table 1 depicts demographic and clinical characteristics of the current sample as well as standardized EF scores. Both groups were comparable in age, sex, years of education and premorbid intelligence. O-LOAD exhibited higher initiation time on the TOL than CS $(p=.044)$ but the other TOL measures showed no difference of statistical relevance. Performance on the Stroop task was lower for O-LOAD compared to CS $(p=.040)$. No additional differences were found for the rest of the EF tasks. Regarding the process-approach analysis of the EF tasks (Table 2), OLOAD showed increased switching errors on the TMT B when compared to CS $(p=.029)$ as well as a greater number of errors on the Stroop task $(p=.050)$. 
Table 3 exhibits both traditional and process scores for the RAVLT. Maximum encoding (Trial A5) and the total learning slope showed better performance in CS ( $p=.030, p=.045$, respectively). O-LOAD also exhibited decreased scores on delayed recall $(p=.003)$ and recognition $(p=.009)$ compared to CS. Regarding the process analysis, O-LOAD showed heightened susceptibility to proactive interference (List $B(p=.006)$, B Interference $E(p=.014)$, Trial $B-A 1(p=.003))$ as well as retroactive interference (Trial $A 6$ Intrusion $E, p=.024$ ).

No significant correlations between Stroop and RAVLT measures were found for CS. However, O-LOAD exhibited positive correlations between de Stroop WordColor trial and the RAVLT's Learning Curve $(r=.437, p=.023)$, List $B(r=.482$, $\mathrm{p}=.009)$, and Delayed Recall $(r=.506, p=.007)$.

\section{Discussion}

This investigation represents an attempt to examine subtle deficits in executive function among middle-age O-LOAD individuals with no cognitive complaints and who were clinically asymptomatic. The main findings of the present study are that

1) O-LOAD subjects exhibited a decreased executive performance compared to CS on the Tower of London (TOL) initiation time and the Stroop Word-Color condition 2) process approach scores of TMT B switching errors and Stroop errors were also greater in O-LOAD than CS; 3) RAVLT learning (Trial A5, Learning Slope), delayed recall and recognition showed a poorer performance on O-LOAD when compared to CS; 4) O-LOAD displayed increased proactive (List B, B 
Interference $E$, Trial B - A1) and retroactive interference (Trial A6 Intrusion E) effects on the RAVLT compared to CS; and 5) positive correlations were found between the Stroop Word-Color trial and the learning curve, List B, and delayed recall trials on the RAVLT only on O-LOAD.

Even though traditionally, a longer initiation time on the TOL reflects planning abilities, the fact that execution and total time showed no significant differences between groups suggests that O-LOAD did not benefit from longer planning time, therefore possibly indicating either an inefficient planning strategy or greater problem-solving difficulties to approach the task at hand. Differences on Stroop performance are more straightforward, evidencing greater susceptibility to interference effects in O-LOAD. This is further supported by the increased number of errors committed on the Stroop test, which are instances where subjects failed to inhibit interfering information.

Another interesting finding when employing the process approach is the fact that even though both groups had a similar performance on the TMT B, O-LOAD participants exhibited more switching errors than CS, likely reflecting early, subclinical difficulties associated to cognitive flexibility processes.

We have already reported O-LOAD's decreased anterograde memory performance (Abulafia et al., 2017), which reflects early subtle cognitive decline in subjects with increased risk of developing AD. The additional findings in this study support our hypothesis that such difficulties may be in part, associated with executive deficits rather than purely mnestic processes as described by the literature which states 
that while the medial temporal lobes mediate memory storage, the frontal lobes are important for the acquisition and encoding of new information, and for the retrieval of the stored data as well as the recollection of source information (Dickerson and Atri, 2014). Our findings of reduced encoding and retrieval scores support this hypothesis. Additionally, proactive and retroactive interference effects during the process of encoding and recalling newly acquired information were observed in OLOAD subjects. These results are in line with previous findings of interference effects on memory tests (Libon et al., 2011; Loewenstein et al., 2017; Curiel et al., 2018; Loewenstein et al., 2018a) which aim at the existence of an interplay between memory and executive processes. In a previous study, our team has reported such effects are already present in asymptomatic middle-aged individuals with family history of AD (Abulafia et al., 2018) providing further support that $E F$ processes such as inhibition of irrelevant information play a key role in memory performance even in the absence of hippocampal atrophy (Duarte-Abritta et al., 2018) and that executive impairment is not only present in all MCls - including the so called "pure" amnestic groups - but also it may also act as a discriminating factor between prodromal $\mathrm{AD}$ and $\mathrm{MCl}$ (Brandt et al., 2009).

The present study has some limitations. Due to the cross-sectional nature of the study we cannot know if our participants will progress to $\mathrm{aMCl}$ and subsequently be diagnosed with LOAD. Thus, we cannot verify if the subtle changes reported herein will be predictive of the eventual development of the disease. Although our focus was on middle-age O-LOAD participants on executive measures that reflect process features, our sample was homogeneous in regards to ethnicity, 
geographical area, culture, and years of education, thus probably limiting generalizability of the results. Additionally, all subjects completed at least 12 years of education - most even have university studies - therefore presenting high cognitive reserve levels which could be preventing the manifestation of greater subtle preclinical changes that would be otherwise present in individuals with lower cognitive reserve. The relatively modest number of participants would require that the present findings be confirmed in larger samples. Nonetheless, the current results have significant implications for understanding the earliest subtle executive changes in LOAD and is worthy of further research.

In sum, process scores such as intrusion errors or interference measures as the ones assessed in the present study are indicative of executive processes underlying other cognitive functions (Lezak et al., 2004). Recently, Curiel and associates (2018) showed that amyloid deposition was associated with semantic intrusion errors on tests of proactive semantic interference among individuals with $\mathrm{aMCl}$ on a cognitive stress test. This further bolsters the argument of the important function of inhibitory processes associated with AD. Process score impairments have been observed earlier than decline in neuropsychological test total scores in preclinical $A D$ and might be useful cognitive markers of preclinical $A D$ (Edmonds et al., 2015). This type of analysis is non-invasive and cost-effective and could help detect individuals at risk for cognitive decline before onset of overt cognitive impairment (Thomas et al., 2018).

\section{Acknowledgements}


CA is a doctoral fellow from CONICET.

\section{Author Contributions}

SMG, DV, and MFV developed the scientific project. SMG evaluated participants.

CA performed neuropsychological evaluations. CA, DV, and LF processed and analyzed neuropsychological data. CA, LF, DL, CBN, RC, and SMG analyzed the results. CA, DV, and SMG wrote the first draft of the manuscript. All authors edited and approved the final manuscript. 


\section{Conflict of interest}

Dr. Charles B. Nemeroff's disclosures are as follows:

Research/Grants:

National Institutes of Health $(\mathrm{NIH})$, Stanley Medical Research Institute

Consulting (last three years):

Xhale, Takeda, Taisho Pharmaceutical Inc., Prismic Pharmaceuticals, Bracket

(Clintara), Total Pain Solutions (TPS), Gerson Lehrman Group (GLG) Healthcare \&

Biomedical Council, Fortress Biotech, Sunovion Pharmaceuticals Inc., Sumitomo

Dainippon Pharma, Janssen Research \& Development LLC, Magstim, Inc., Navitor

Pharmaceuticals, Inc., TC MSO, Inc., Intra-Cellular Therapies, Inc.

Stockholder:

Xhale, Celgene, Seattle Genetics, Abbvie, OPKO Health, Inc., Network Life Sciences Inc., Antares, BI Gen Holdings, Inc.

Scientific Advisory Boards:

American Foundation for Suicide Prevention (AFSP), Brain and Behavior Research

Foundation (BBRF) (formerly named National Alliance for Research on Schizophrenia and Depression [NARSAD]), Xhale, Anxiety Disorders Association of America (ADAA), Skyland Trail, Bracket (Clintara), RiverMend Health LLC, Laureate Institute for Brain Research, Inc.

Board of Directors: 
AFSP, Gratitude America, ADAA

Income sources or equity of $\$ 10,000$ or more:

American Psychiatric Publishing, Xhale, Bracket (Clintara), CME Outfitters, Takeda

Patents:

Method and devices for transdermal delivery of lithium (US 6,375,990B1)

Method of assessing antidepressant drug therapy via transport inhibition of monoamine neurotransmitters by ex vivo assay (US 7,148,027B2)

Speakers Bureau:

None

All other authors have nothing to disclose nor have any potential conflicts of interest.

\section{Role of funding source}

The present study was supported by a grant from Agencia de Promoción, FONCYT, MINCYT, Argentina (PICT-2014-0633). 


\section{References}

Abulafia, C., Duarte-Abritta, B., Villarreal, M.F., et al., 2017. Relationship between cognitive and sleep-wake variables in asymptomatic offspring of patients with late-onset alzheimer's disease. Front Aging Neurosci. 9, 93.

Abulafia, C., Loewenstein, D., Curiel-Cid, R., et al., 2018. Brain Structural and Amyloid Correlates of Recovery From Semantic Interference in Cognitively Normal Individuals With or Without Family History of Late-Onset Alzheimer's Disease. J Neuropsych Clin N. p.appineuropsych17120355.

Albert, M.S., 1996. Cognitive and neurobiologic markers of early Alzheimer disease. P Natl Acad Sci USA. 93 (24), 13547-13551.

Ballard, C., O'Sullivan, M.J., 2013. Alzheimer disease and stroke: Cognitive and neuroimaging predictors of $A D$ and stroke. Nat Rev Neurol. 9, 605-606.

Baudic, S., Dalla Barba, G., Thibaudet, M.C., et al., 2006. Executive function deficits in early Alzheimer's disease and their relations with episodic memory. Arch Clin Neuropsych. 21 (1), 15-21.

Beck, A.T., Steer, R.A., Brown, G.K., 1996. Beck depression inventory-II. Psychological Corporation. San Antonio, TX.

Binetti, G., Magni, E., Padovani, A., et al., 1996. Executive dysfunction in early Alzheimer's disease. J Neurol Neurosur Ps. 60 (1), 91-93. 
Blacker, D., Lee, H., Muzikansky, A., et al., 2007. Neuropsychological measures in normal individuals that predict subsequent cognitive decline. Arch Neurol. 64 (6), 862 871.

Blanco Martín, E., Ugarriza Serrano, I., Elcoroaristizabal Martín, X., et al., 2016.

Dysexecutive syndrome in amnesic mild cognitive impairment: A multicenter study. BMC Neurol. 16, 1-8.

Brandt, J., Ph, D., Aretouli, E., et al., 2009. Selectivity of executive function deficits in mild cognitive impairment. Neuropsychology. 23, 607-618.

Christidi, F., Kararizou, E., Triantafyllou, N., et al., 2015. Derived Trail Making Test indices: demographics and cognitive background variables across the adult life span. Aging Neuropsychol C. 22 (6), 667-678.

Culbertson, W.C., Zillmer, E., 2001. Tower of London-Drexel University (TOLDX). MultiHealth Systems. North Tonawanda, NY.

Curiel, R., Crocco, E., Czaja, S., et al., 2013. Deficits in semantic cuing, proactive and retroactive interference as early features of Alzheimer's disease. Alzheimers Dement. 9 (4), P456.

Curiel, R.E., Loewenstein, D.A., Rosselli, M., et al., 2018. Semantic Intrusions and Failure to Recover From Semantic Interference in Mild Cognitive Impairment:

Relationship to Amyloid and Cortical Thickness. Curr Alzheimer Res. 15 (9), 848-855.

Delis, D.C., Kaplan, E., Kramer, J.H., 2001. Delis-Kaplan executive function system: Technical manual. Psychological Corporation. New York, NY. 
Dickerson, B.C., Atri, A., 2014. Dementia: comprehensive principles and practice. Oxford University Press. UK.

Donix, M., Ercoli, L.M., Siddarth, P., et al., 2012. Influence of alzheimer disease family history and genetic risk on cognitive performance in healthy middle-aged and older people. American J Geriat Psychiat. 20, 565-573.

Duarte-Abritta, B., Villarreal, M.F., Abulafia, C., et al., 2018. Cortical thickness, brain metabolic activity, and in vivo amyloid deposition in asymptomatic, middle-aged offspring of patients with late-onset Alzheimer's disease. J Psychiatr Res. 107, 11-18.

Edmonds, E.C., Delano-Wood, L., Galasko, D.R., et al., 2015. Subtle Cognitive Decline and Biomarker Staging in Preclinical Alzheimer's Disease. J Alzheimers Dis. 47, 231242.

Folstein, M.F., Folstein, S.E., McHugh, P.R., 1975. "Mini-mental state". A practical method for grading the cognitive state of patients for the clinician. J Psychiatr Res. 12 (3) 189-198.

Golden, C.J., 1978. Stroop color and word test: a manual for clinical and experimental uses. Stoelting. Chicago, IL.

Hazlett, K.E., Figueroa, C.M., Nielson, K.A., 2015. Executive functioning and risk for alzheimer's disease in the cognitively intact: Family history predicts Wisconsin card sorting test performance. Neuropsychology. 29, 582-591. 
Houston, W.S., Delis, D.C., Lansing, A., et al., 2005. Executive function asymmetry in older adults genetically at-risk for Alzheimer's disease: Verbal versus design fluency. $J$ Int Neuropsych Soc, 2005. 11, 629-642.

Jaeger, R.G., Halliday, T.M., 1998. On Confirmatory versus Exploratory Research. Herpetologica. 54, S64-S66.

Johns, E.K., Phillips, N.A., Belleville, S., et al., 2012. The profile of executive functioning in amnestic mild cognitive impairment: disproportionate deficits in inhibitory control. J Int Neuropsych Soc. 18 (3), 541-555.

Jurado, M.B., Rosselli, M., 2007. The elusive nature of executive functions: A review of our current understanding. Neuropsychol Rev. 17, 213-233.

Kaplan, E., 1988. The process approach to neuropsychological assessment. Aphasiology. 2 (3-4), 309-311.

Kirova, A.M., Bays, R.B., Lagalwar, S., 2015. Working memory and executive function decline across normal aging, mild cognitive impairment, and Alzheimer's disease. BioMed Res Int. 748212.

Lezak, M.D., Howieson, D.B., Loring, D.W., et al., 2004. Neuropsychological assessment. Oxford University Press, USA.

Libon, D.J., Bondi, M.W., Price, C.C., et al., 2011. Verbal serial list learning in mild cognitive impairment: A profile analysis of interference, forgetting, and errors. J Int Neuropsych Soc. 17, 905-914. 
Loewenstein, D.A., Curiel, R.E., DeKosky, S., et al., 2018a. Utilizing semantic intrusions to identify amyloid positivity in mild cognitive impairment. Neurology. 91 (10), 976-984.

Loewenstein, D.A., Curiel, R.E., Duara, R., et al., 2018b. Novel Cognitive Paradigms for the Detection of Memory Impairment in Preclinical Alzheimer's Disease. Assessment. 25, 348-359.

Loewenstein, D.A., Curiel, R.E., Wright, C., et al., 2017. Recovery from Proactive Semantic Interference in Mild Cognitive Impairment and Normal Aging: Relationship to Atrophy in Brain Regions Vulnerable to Alzheimer's Disease. J Alzheimers Dis. 56, $1119-1126$.

McDonald, J., 2014. Handbook of Biological Statistics. 3rd Ed. Sparky House Publishing. Baltimore, MD.

Moro, V., Condoleo, M.T., Valbusa, V., et al., 2015. Cognitive stimulation of executive functions in mild cognitive impairment: specific efficacy and impact in memory. Am $\mathrm{J}$ Alzheimers Dis. 30 (2), 153-164.

Rapp, M.A., Reischies, F.M., 2005. Attention and executive control predict Alzheimer disease in late life: results from the Berlin Aging Study (BASE). American J Geriat Psychiat. 13 (2), 134-141.

Reiner, A., Yekutieli, D., Benjamini, Y., 2003. Identifying differentially expressed genes using false discovery rate controlling procedures. Bioinformatics. 19 (3), 368-375.

Reinvang, I., Grambaite, R., Espeseth, T., 2012. Executive dysfunction in MCI: Subtype or early symptom. Int J Alzheimers Dis. 2012. 
Reinvang, I., Winjevoll, I.L., Rootwelt, H., et al., 2010. Working memory deficits in healthy APOE epsilon 4 carriers. Neuropsychologia. 48 (2), 566-573.

Reitan, R.M., Wolfson, D., 1985. The Halstead-Reitan neuropsychological test battery: Theory and clinical interpretation. Reitan Neuropsychology.

Rey, A., 1964. L'examen clinique en psychologie. Presses Universitaires de France. Paris.

Rosen, W.G., Terry, R.D., Fuld, P.A., et al., 1980. Pathological verification of ischemic score in differentiation of dementias. Ann Neurol. 7 (5), 486-488.

Salmon, D., Bondi, M., 2009. Neuropsychological Assessment of Dementia, Annu Rev Psychol. 60, 257-282.

Sánchez, S.M., Abulafia, C., Duarte-Abritta, B., et al., 2017. Failure to Recover from Proactive Semantic Interference and Abnormal Limbic Connectivity in Asymptomatic, Middle-Aged Offspring of Patients with Late-Onset Alzheimer's Disease. J Alzheimers Dis. $60,1183-1193$.

Schmidt, M., 1996. Rey auditory verbal learning test: A handbook. Western Psychological Services. Los Angeles, CA.

Sierra Sanjurjo, N., Montañes, P., Sierra Matamoros, F.A., et al., 2015. Estimating intelligence in Spanish: regression equations with the Word Accentuation Test and demographic variables in Latin America. Appl Neuropsych-Adul. 22 (4), 252-261.

Spreen, O., Benton, A.L., 1977. Neurosensory center comprehensive examination for aphasia. Neuropsychological Laboratory. Victoria, BC. 
Strauss, E., Sherman, E.M., Spreen, O., 2006. A compendium of neuropsychological tests: Administration, norms, and commentary. 3rd ed. Oxford University Press. New York, NY.

Swanberg, M.M., Tractenberg, R.E., Mohs, R., et al., 2004. Executive dysfunction in Alzheimer disease. Arch Neurol-Chicago. 61 (4), 556-560.

Thomas, K.R., Eppig, J., Edmonds, E.C., et al., 2018. Word-list intrusion errors predict progression to mild cognitive impairment. Neuropsychology. 32, 235-245.

Troyer, A.K., Moscovitch, M., Winocur, G., 1997. Clustering and switching as two components of verbal fluency: evidence from younger and older healthy adults. Neuropsychology. 11 (1), 138-146.

Vik, P., Ruff, R.R., 1988. Children's figural fluency performance: Development of strategy use. Dev Neuropsychol. 4 (1), 63-74.

Wechsler, D., 1997. Wechsler adult intelligence scale-III. The Psychological Corporation. San Antonio, TX.

Wetter, S.R., Delis, D.C., Houston, W.S., et al., 2005. Deficits in inhibition and flexibility are associated with the APOE-E4 allele in nondemented older adults. J Clin Exp Neuropsyc. 27 (8), 943-952.

Zheng, D., Dong, X., Sun, H., et al., 2012. The overall impairment of core executive function components in patients with amnestic mild cognitive impairment: a crosssectional study. BMC Neurol. 12 (1), 138. 